\title{
INTERSTELLAR EXTINCTION IN EXTERNAL GALAXIES
}

\author{
EDWARD L. FITZPATRICK \\ Princeton University Observatory
}

\begin{abstract}
In this paper I review the existing measurements of the wavelength dependence of interstellar extinction arising from dust situated outside the Milky Way galaxy. This paper emphasizes studies of extinction in the Large and Small Magellanic Clouds. I discuss the UV extinction properties, as well as the optical and near-IR properties as determined from ground-based photometry.
\end{abstract}

\section{INTRODUCTION}

In this paper, I review the current status of observations of interstellar extinction arising from dust outside of the Milky Way. The emphasis will be on results for the Large and Small Magellanic Clouds (LMC and SMC), which are by far the best-studied extragalactic systems. In addition, and betraying my own bias, I will concentrate on ultraviolet (UV) extinction results obtained with the International Ultraviolet Explorer (IUE) satellite and only briefly discuss optical and near-IR results derived from ground-based observations. The theoretical interpretation of these observations will be left for other authors.

Since this entire volume is devoted to studies of interstellar dust, and since most readers will be similarly devoted, I do not need to dwell on the obvious point that the study of interstellar extinction is the single most important topic in modern astronomy (!). I will note, however, that the motivations for investigating extragalactic extinction are essentially identical to those for studying Galactic extinction. The wavelength dependence of extinction, when properly interpreted through theory, yields quantitative measures of the composition and size distribution of interstellar grains. Such results are required for assessing the importance of dust grains in interstellar heating/cooling, interstellar chemistry, etc.. A knowledge of the extinction properties is also required for deriving the intrinsic continua shapes of extragalactic objects, such as hot stars in the Magellanic Clouds.

This paper is organized by galaxy. The extinction properties of the LMC are considered in $\S 2$, those of the SMC in $\S 3$, and those for several other, more poorly studied, galaxies in $\S 4$. 


\section{THE LARGE MAGELLANIC CLOUD}

\subsection{UV EXTINCTION}

With the launch of the $I U E$ satellite in 1978 , it became possible to measure directly the wavelength dependence of LMC UV extinction via the "pair method," i.e., by taking the ratio of the UV fluxes of a reddened star, located behind or within a dust cloud, with the fluxes of an identical, but unreddened, comparison star. This technique has revealed a wealth of new information in recent years on the properties of extinction in the Milky Way (see the review by Massa and Savage, 1989), and has been similarly rewarding for the LMC.

In Galactic extinction studies, the most desirable types of stars to use with the pair method are the late-O and early- $B$ main sequence stars, which have wellunderstood and well-determined intrinsic continuum shapes (see, e.g., Massa, Savage, and Fitzpatrick, 1983). For the LMC, the distance modulus of about 18.5 mag makes these stars very difficult to observe, even in the low-resolution mode of IUE. Instead, the OB Ia supergiants, which are the brightest normal stars in the UV spectral region (and have visual magnitudes in the range $V=10.5-12.5$ ), are used to form reddening pairs (i. e., the combination of a reddened star plus its comparison star). Spectra of most LMC supergiants can be obtained easily in the low-resolution IUE mode. The LMC has many very lightly reddened supergiants - in contrast to the Milky Way - so that appropriate comparison stars exist for most reddened stars. A potential problem, however, is that the spectral types for LMC stars are generally poorly determined and other information may have to be used to identify good reddening pairs. In addition, the typical $E(B-V)$ values for the LMC stars are small $(<0.2 \mathrm{mag})$, so that errors introduced by mismatch of reddening pairs and by photometric uncertainties are greatly magnified in the production of the standard, normalized extinction curves.

The first major IUE studies of LMC extinction were performed by Koornneef and Code' (1981, hereafter KC) and Nandy et al. (1981, hereafter NMW; see also Nandy and Morgan, 1978; and Nandy et al., 1980). KC and NMW used observations of 8 and 14 reddened supergiants, respectively, with an overlap of 4 stars. The average extinction curves from each of these studies are shown in Figure 1. Both found a steeper far-UV rise and a much weaker $2175 \AA$ bump than in the average Milky Way extinction curve (heavy solid line in Fig. 1). NMW reported no evidence for regional variations in the LMC extinction properties (except for the star Sk-69 108 which yielded a Galactic-type curve, see Nandy et al., 1980). KC's sample was concentrated near the 30 Doradus nebula (hereafter " 30 Dor") in the LMC and could not be used to comment on regional variations. Nine of the 14 stars in the NMW sample are also located very near to 30 Dor. A more extensive review of this early work is given by Nandy (1984).

The KC and NMW curves are often referred to as the "average LMC extinction curves." They are used to deredden the energy distributions of stars located in all regions of the LMC and it is frequently stated that these curves demonstrate the effects of lower-than-solar metallicities on dust extinction properties. In the remainder of this section, I hope to discourage both practices!

An indication that LMC extinction properties are more complex than suggested by the KC and NMW studies came from the work of Clayton and Martin (1985; 


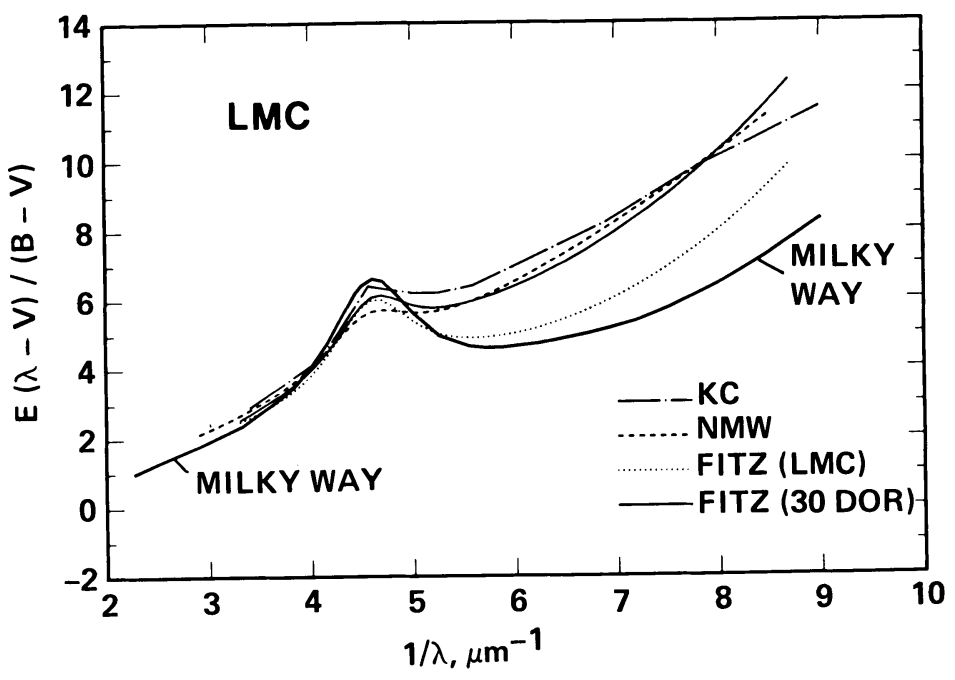

Fig. 1. Large Magellanic Cloud (LMC) extinction curves. $\mathrm{KC}=$ Koornneef and Code (1981), NMW = Nandy et al. (1981), Fitz = Fitzpatrick.

hereafter CM). For their total sample of 12 stars, CM found a mean curve resembling the $\mathrm{KC}$ and $\mathrm{MNW}$ results but with a systematic difference between the mean extinction properties of the 6 stars located within about $1^{\circ}$ of 30 Dor and the 6 stars located in other LMC regions. The far-UV portion of the 30 Dor region curve $(\lambda<1700 \AA)$ was about 2 units higher in $E(\lambda-V) / E(B-V)$ than the non-30 Dor curve. The CM and NMW studies share 6 reddened stars in common and the different conclusions on regional variations of LMC extinction were surprising.

In order to recognize real extinction variations it is, of course, necessary to quantify the uncertainties in extinction curves. The potential errors include those arising from photometric uncertainties and from spectral mismatch of reddened and comparison stars. A particular problem affecting both CM and NMW is systematic mismatch error, resulting from inaccurate spectral classifications. In both studies, there is a strong tendency for the reddened supergiants to be paired with hotter, fainter comparison stars. For example, in the NMW sample the typical comparison star has an absolute magnitude nearly 2 magnitudes fainter than the typical reddened star. This introduces mismatch in both the strong stellar spectral features and in the general continuum shape. With the resultant extinction curves strongly modulated by mismatch, correctly identifying intrinsic variations may be very difficult.

The issue of regional variations was considered in the most recent LMC extinction study, carried out by the author (Fitzpatrick 1985a and 1986, hereafter Paper I and Paper II). Two of the goals of this study were to eliminate spectral mismatch as a significant factor in the analysis (through the observation of a large grid of unreddened LMC supergiants) and to quantify the uncertainties due to photomet- 
ric error. Both of these issues, and particularly the method for choosing appropriate reddening pairs, are discussed in detail in Paper $\mathrm{I}$.

The sample of reddened stars in Papers I and II consists of 27 supergiants; 11 stars were taken from previous studies and 16 were newly observed. Stars from earlier studies were rejected for various reasons, including stellar peculiarities, lack of a suitable comparison star, or too small an $E(B-V)$ value. As had been done by previous investigators, the extinction properties were considered for 2 regional groupings: the 30 Doradus group, consisting of 12 stars located within $0.5^{\circ}$ of the core of 30 Dor, and the non-30 Doradus group, consisting of 15 stars located in a variety of LMC locations farther than about $1^{\circ}$ from 30 Dor.

For the 30 Dor group, I concluded in Paper I that the apparently large scatter seen among the 12 curves was as expected from uncertainties in the optical and IUE photometry. The expected scatter is large because the uncertainties in an extinction curve scale as $\sigma[E(B-V)] / E(B-V)$, and $\sigma[E(B-V)]$ is relatively large compared to $E(B-V)$ in the LMC ( 0.02 vs. $\sim 0.16$, respectively). The measurements are thus consistent with a single UV extinction "law" in the general region of radius $0.5^{\circ}-1.0^{\circ}$ around the 30 Dor nebula. The average curve for this region is shown by the thin solid line in Figure 1 [labelled "Fitz (30 Dor)"]. It is essentially identical to the NMW and KC curves (which were derived mainly from 30 Dor stars), with a steep far-UV rise and very weak $2175 \AA$ bump.

Outside the 30 Dor region the results are not so straightforward. Two of the 15 non-30 Dor stars clearly stood out from the group in having extremely steep far-UV extinction curves (see Fig. 3 in Paper II). These stars deserve further attention to determine if the apparently extreme extinction is due to some unrecognized stellar peculiarity. The remaining 13 stars showed a small scatter in their far-UV levels, which was consistent with the scatter expected from photometric uncertainties. The scatter in the $2175 \AA$ bump region, however, indicated a true intrinsic variation. An average curve was constructed from these 13 curves and is shown in Figure 1 [labelled "Fitz (LMC)"]. This curve has a significantly lower far-UV level and a stronger average $2175 \AA$ bump than the 30 Dor curves. It was presented in Paper II as a better representative of "typical" extinction in the LMC than the curves derived from stars near 30 Dor.

One of the main conclusions from Paper II was, therefore, that typical UV extinction in the LMC - outside of a restricted region around the 30 Dor nebula - is much more similar to the Milky Way average than has been considered in the past. The KC and NMW curves, which are strongly weighted to stars in the 30 Dor region, are not representative of the $\mathrm{LMC}$ as a whole and cannot necessarily be held as examples of the effects of lower metallicity on the extinction. Properties particular to the 30 Dor region, rather than to the LMC in general, are responsible for the extreme far-UV extinction and perhaps the weak $2175 \AA$ bump seen in the 30 Dor curves. The average LMC curve in Figure 1 should be viewed with the same caution as should be applied to average Milky Way curves. It is the mean of curves which show intrinsically different bump strengths and, therefore, may not be representative of the extinction in any particular line of sight. Also, sincc the observational errors are large, some level of intrinsic variation in the far-UV is almost certainly present, but buried in the noise. The very different extinction curves seen toward 2 of the 15 non-30 Dor stars, if confirmed, may indicate very significant regional variations outside the 30 Dor region. New observations are 
required to examine spatial variations on a finer scale than the coarse 30 Dor vs. non-30 Dor comparison.

For correcting LMC stellar energy distributions, a regional approach should be followed. Stars within about $0.5-1^{\circ}$ of 30 Dor should be corrected with the 30 Dor regional curve (or the KC or NMW curves). Stars outside the 30 Dor region should be corrected with the "LMC" curve in Figure 1 . This will make the best possible correction to the far-UV flux levels, but may over- or under-correct the bump region, due to the variations seen in the bump strength. Because of these variations, correcting energy distributions by "ironing out" the bump may give very erroneous results.

The LMC and 30 Dor extinction curves produced in Papers I and II were fitted with an analytical function, derived by Fitzpatrick and Massa (1988) for Galactic curves. (It is these fits which are shown in Figure 1.) The function and the best fit parameters for reproducing the curves are given in Paper II. The region around the $2175 \AA$ bump is fitted with a Lorentzian-like function which yields three parameters, which describe the strength of the bump, the location of the peak, and the FWHM. This function reproduces the shapes of the LMC bumps as accurately as for the Galactic curves. The bump peaks are located very close to the average of the Fitzpatrick and Massa sample, and the FWHM's are within the range observed for the Galaxy. The LMC and 30 Dor average curves have bumps which are $80 \%$ and $50 \%$ as strong, respectively, as the average of the Galactic sample. These results suggest that the LMC bumps are produced by the same type(s) of grains and the same physical process(es) as in the Milky Way, although the relative numbers of such grains may differ. This is not necessarily a surprising result, but it is reassuring.

\subsection{OPTICAL/IR EXTINCTION AND THE GAS-TO-DUST RATIO}

Very little work on the properties of optical extinction in LMC has been done, outside of measurements in the UBV system. Most recently, Papers I and II have shown that the ratio of $E(U-B) / E(B-V)$ differs between the 30 Dor region and the other LMC regions. A ratio of $0.89 \pm 0.08$ was found for the 30 Dor stars and a ratio of $0.75 \pm 0.04$ for the stars outside 30 Dor. The "standard value" generally quoted for the Milky Way is $\mathbf{0 . 7 2}$.

The near-IR extinction law in the LMC has been examined by Koornneef (1982), Morgan and Nandy (1982) and CM, all using JHK photometry and some version of the pair method. The stars used were essentially the same as used in the UV studies of the respective authors. All the studies found values for $R\left[\equiv A_{V} / E(B-V)\right]$ not significantly different from the average Milky Way value of 3.1. The spectral mismatch problems present in the UV studies, however, are also present at some level in the IR analyses. It would be very worthwhile to acquire near-IR photometry of the reddened and unreddened stars from Papers I and II (very few of which have been observed) to eliminate mismatch effects in the IR results. Linear polarization measurements by Clayton, Martin, and Thompson (1983) also yield Galactic-like $R$ values, assuming that the same relation between $\lambda_{\max }$ and $R$ exists in both galaxies.

The LMC gas-to-dust ratio was reviewed by Koornneef (1984). Results from IUE measurements of the HI column densities have been published by Koornneef (1982) and in Papers I and II. Koornneef found $N(H I) / E(B-V)=2 \cdot 10^{22} \mathrm{~cm}^{-2} \mathrm{mag}^{-1}$ for the $\mathrm{KC}$ extinction sample - which is dominated by stars in the 30 Dor region. A 
nearly identical result, $2.4 \cdot 10^{22} \mathrm{~cm}^{-2} \mathrm{mag}^{-1}$, was reported in Paper I for the 30 Dor region. These ratios are about 5 times greater than the Galactic average measured by Bohlin (1975). The gas-to-dust ratio outside the 30 Dor region was found in Paper II to range between the Galactic and 30 Dor values.

\section{THE SMALL MAGELLANIC CLOUD}

\subsection{UV EXTINCTION}

The measurement of extinction properties in the SMC provides similar observational challenges as for the LMC. The distance modulus is about half-a-magnitude fainter than the LMC's modulus, so that the OB supergiants, which are still easily within the capabilities of the low-resolution cameras of $I U E$, must be used to form reddening pairs. However, the SMC has a much smaller massive star population than the LMC, so the choice of stars is more restricted - and finding an appropriate comparison star may be more difficult. Most SMC spectral types are even more poorly determined than for LMC stars, which also complicates the selection of appropriate reddening pairs. Additionally, the SMC has a lower dust content than the LMC, so the typical reddening is much lower. Since the uncertainties in individual extinction curves scale inversely with $E(B-V)$, a typically reddened SMC star [with $E(B-V)<0.1$ ] can yield only a very uncertain extinction curve, even if an accurate comparison star can be found. As a result, current SMC extinction results depend on a very small number of stars, which have unusually large reddenings. This leads to the question of whether their extinction properties can be considered representative of the SMC as a whole.

UV extinction measurements for the SMC using IUE data have been reported by Rocca-Volmerange et al. (1981), Nandy et al. (1982), Hutchings (1982), Lequeux et al. (1982), Bromage and Nandy (1983), and Prévot et al. (1984). The best determined result from these studies is shown in Figure 2, which is adapted from the paper by Prévot et al.. The extinction curve which has become accepted as typical of the SMC (solid line in Fig. 2) is nearly linear, in $\lambda^{-1}$, from the optical region through the far-UV. The familiar $2175 \AA$ bump is absent and the far-UV level is more extreme than even the 30 Doradus region of the LMC (dashed line in Fig. 2).

The interpretation of the Prévot et al. curve in Figure 2 as representative of the SMC in general has to be viewed with caution, since it is based on only 3 stars, which have atypically large reddenings. (The curve produced by Bromage and Nandy is derived from a larger number of stars but, for the reasons given by Prévot et al., cannot be considered better determined.) Prévot et al. note the existence of 2 other SMC stars, Sk124 and Sk143, with very different extinction curves. Sk124 yields an extinction curve more similar to the 30 Dor curves, although since this star is a rather peculiar $\sim$ B3 supergiant - with Balmer emission and an $H \beta$ P Cygni profile (from digital spectra obtained at Cerro Tololo Observatory by the author) - it's extinction curve is considered uncertain. Sk143, on the other hand, is an apparently normal 09 I: star (also from CTIO spectra) which yields a Galactic-like extinction curve. This curve cannot result from spectral mismatch effects or photometry errors and clearly represents a regional variation in SMC extinction properties - and a potentially enormous range in the possible levels of far-UV extinction in the SMC. 


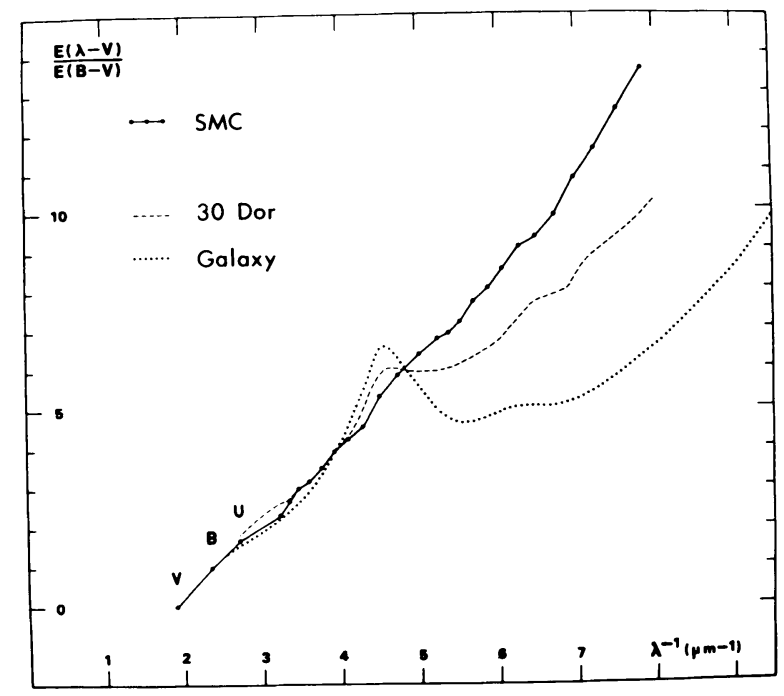

Fig. 2. The typical Small Magellanic Cloud (SMC) extinction curve. Adapted from Prévot et al., 1984.

Further work on SMC extinction is obviously needed to define the mean properties and the possible range of variation. It is not clear that there are many more potential IUE targets to be observed, however, and a better understanding of SMC extinction may have to wait for more sensitive UV spectrometers. Until then, the dereddening of SMC stellar energy distributions will be an extremely uncertain process.

\subsection{OPTICAL/IR EXTINCTION AND THE GAS-TO-DUST RATIO}

UBV measurements by Bouchet et al. (1985) yield an optical extinction ratio for a group of SMC stars of $E(U-B) / E(B-V) \simeq 0.81$. This value seems to me to be rather small if the very steep UV extinction law is widespread in the SMC. In the Galactic extinction sample studied by Fitzpatrick and Massa (1988) the stars with steep far-UV extinction also tend strongly to have larger $E(U-B) / E(B-V)$ ratios. This is consistent with the value of 0.89 found for the steep curve in the 30 Dor region. From this point of view, the SMC stars would be expected to have optical reddening ratios of about 1.0. The large measurement uncertainties may make this a difficult number to determine.

Near-IR extinction measurements have been performed by Nandy, Morgan, and Houziaux (1984) and Bouchet et al. (1985). Both groups find results similar to those in the Milky Way, with $R \simeq 3$. From a statistical analysis of SMC UBV data, Isserstedt (1980) suggests a value of $R \simeq 2$. The recent finding by Cardelli, Clayton, and Mathis (1988) of a correlation between $R$ and the far-UV extinction properties in a sample of Galactic stars would suggest a smaller-than-Galactic value for $\boldsymbol{R}$ 
in the SMC. Some of the first polarization results for SMC stars were reported by Magalhães et al. at this meeting (Magalhães et al., 1989). The extension of this study to a larger sample of stars will certainly be useful for investigating the properties of SMC dust grains and, perhaps, for pinning down the value of $R$ in the SMC.

Fitzpatrick (1985b) and Bouchet et al. (1985) both examined the SMC gas-todust ratio using a very similar sample of $\sim 20$ SMC stars with low-dispersion IUE spectra. Both studies found the ratio $N(H I) / E(B-V)$ to be at least 10 times greater than the Galactic average. Interestingly, the star Sk143, which yields a Galactic-like UV extinction curve, also yields a Galactic gas-to-dust ratio. If the physical conditions which produce the very large gas-to-dust ratio also produce a steep UV extinction law, these measurements may indicate that the Prévot et al. extinction curve - while based on only 3 stars - really does represent the typical UV extinction properties in the SMC.

\section{OTHER GALAXIES}

Beyond the Magellanic Clouds, the measurement of extinction becomes very difficult. In M31 and M33, the next nearest large galaxies, the normal OB supergiants have $V$ magnitudes in the range 17-18 mag. Only a few are within the capabilities of $I U E$, making large scale pair method studies impractical. In addition, optical surveys are still needed to identify and classify the normal stars, as has been done for the Magellanic Clouds.

Some attempts have been made to infer extinction properties from less-thannormal objects in M31 and M33 (e.g., Massey and Hutchings 1983; D'Odorico and Benvenuti 1983; Massey, Hutchings, and Bianchi, 1985; and Hutchings, Massey, and Bianchi, 1987). These authors have generally concluded that the UV extinction is due to an "LMC-type" extinction law, by which they mean the 30 Dor curve, with a steep far-UV and weak $2175 \AA$ bump. The problem with these studies is that it is not always clear exactly what kind of object is being observed or what it's intrinsic energy distribution (which is required to derive the extinction) ought to be. For example, Hutchings, Massey, and Bianchi (1987) state that the "LMCtype" extinction curve is required to understand the observed energy distribution of a M31 "O6" star. In fact, this star is an early B-type supergiant, whose energy distribution may be understood easily using a Galactic extinction law.

A recent paper by Bohlin et al. (1988) discusses rocket-UV observations of globular clusters in M31. These authors conclude that the broad-band near-UV (2700 $\AA)$ and far-UV $(1500 \AA)$ colors of the clusters can be understood with a Galactic extinction law.

M31 and M33 are probably the best potential targets for future pair method extinction studies using reasonably normal stars. In other galaxies crowding problems will require that extinction information be gathered from cluster of stars or from very luminous peculiar objects. At present, such information consists chiefly of comments on whether the $2175 \AA$ bump is present in $I U E$ spectra of these objects. See, e.g., the discussion by Walsh, Nandy, Thompson, and Meaburn (1986) of the UV spectra of the "hot spot" galaxies NGC1097 and NGC2997. 
ACKNOWLEDGEMENTS. This paper is dedicated to the memory of my friend Tom Kukucka. He was a helluva good guy.

\section{REFERENCES}

Bohlin, R. C. 1975, Ap. J., 200, 402.

Bohlin, R. C., Cornett, R. H., Hill, J. K., Hill, R. S., and Stecher, T. P. 1988, Ap. J., 384, in press.

Bouchet, P., Lequeux, J., Maurice, E., Prévot, L., and Prévot-Burnichon, M. L. 1985, Astr. Ap., $149,330$.

Bromage, G. E., and Nandy, K. 1983, M. N. R. A. S., 204, 29P.

Cardelli, J., Clayton, G. C., and Mathis, J. M. 1988, Ap. J. (Letters), 329, L33.

Clayton, G. C., Martin, P. G., and Thompson, I. 1983, Ap. J., 265, 194.

Clayton, G. C., and Martin, P. G. 1985, Ap. J., 288, 558 (CM).

D'Odorico, S., and Benvenuti, P. 1983, M. N. R. A. S., 203, 157.

Fitzpatrick, E. L. 1985a, Ap. J., 299, 219 (Paper I).

1985b, Ap. J. Suppl., 59, 77.

- 1986, A. J., 92, 1068 (Paper II).

Fitzpatrick, E. L., and Massa, D. 1988, Ap. J., 328, 734.

Hutchings, J. B. 1982, Ap. J., 255, 70.

Hutchings, J. B., Massey, P., and Bianchi, L. 1987, Ap. J. (Letters), 322, L79.

Isserstedt, J. 1980, Astr. Ap., 83, 322.

Koornneef, J. 1982, Astr. Ap., $107,247$.

1984, in IAU Symposium 108, Structure and Evolution of the Magellanic Clouds, eds. S. van den Bergh and K. S. de Boer, (Dordrecht: Reidel), p. 333.

Koornneef, J., and Code, A. D. 1981, Ap. J., 247860 (KC).

Lequeux, J., Maurice, E., Prévot-Burnichon, M. L., Prévot, L., and Rocca-Volmerange, B. 1982, Astr. Ap., 113, L15.

Magalhães, A. M., Piirola, V., Coyne, G. V., Rodrigues, C. V. 1989, in Interstellar Dust Contributed Papers, eds. A. G. G. M. Tielens and L. J. Allamandola, NASA CP-3036.

Massa, D., Savage, B. D., and Fitzpatrick, E. L. 1983, Ap. J., 266, 662.

Massa, D., and Savage, B. D. 1989, in IAU Symposium 195, Interstellar Dust, eds., L. J. Allamandola and A. G. G. M. Tielens, (Dordrecht: Kluwer), p. 3.

Massey, P., and Hutchings, J. B. 1983, Ap. J., 275, 578.

Massey, P., Hutchings, J. B., and Bianchi, L. 1985, A. J., 90, 2239.

Morgan, D. H., and Nandy, K. 1982, M. N. R. A. S., 199, 979.

Nandy, K. 1984, in IAU Symposium 108, Structure and Evolution of the Magellanic Clouds, eds. S. van den Bergh and K. S. de Boer, (Dordrecht: Reidel), p. 341.

Nandy, K., and Morgan, D. H. 1978, Nature, 276, 478.

Nandy, K., Morgan, D. H., Willis, A. J., Wilson, R., Gondehalekar, P. M., and Housiaux, L. 1980, Nature, 283, 725.

Nandy, K., Morgan, D. H., Willis, A. J., Wilson, R., and Gondhalekar, P. M. 1981, M. N. R. A. S., 196, 955 (NMW).

Nandy, K., McLachlan, A., Thompson, G. I., Morgan, D. H., Willis, A. J., Wilson, R., Gondhalekar, P. M., and Houziaux, L. 1982, M. N. R. A. S., 201, iP.

Nandy, K., Morgan, D. H., and Houziaux, L. 1984, M. N. R. A. S., 211, 895.

Prévot, M. L., Lequeux, J., Maurice, E., Prévot, L., and Rocca-Volmerange, B. 1984, Astr. Ap., $182,389$.

Volmerange, B., Prévot, L., Ferlet, R., Lequeux, J., and Prévot-Burnichon, M. L. 1981, Astr. Ap., $99, \mathrm{~L} 5$.

Walsh, J. R., Nandy, K., Thompson, G. I., and Meaburn, J. 1986, M. N. R. A. S., 220, 453. 


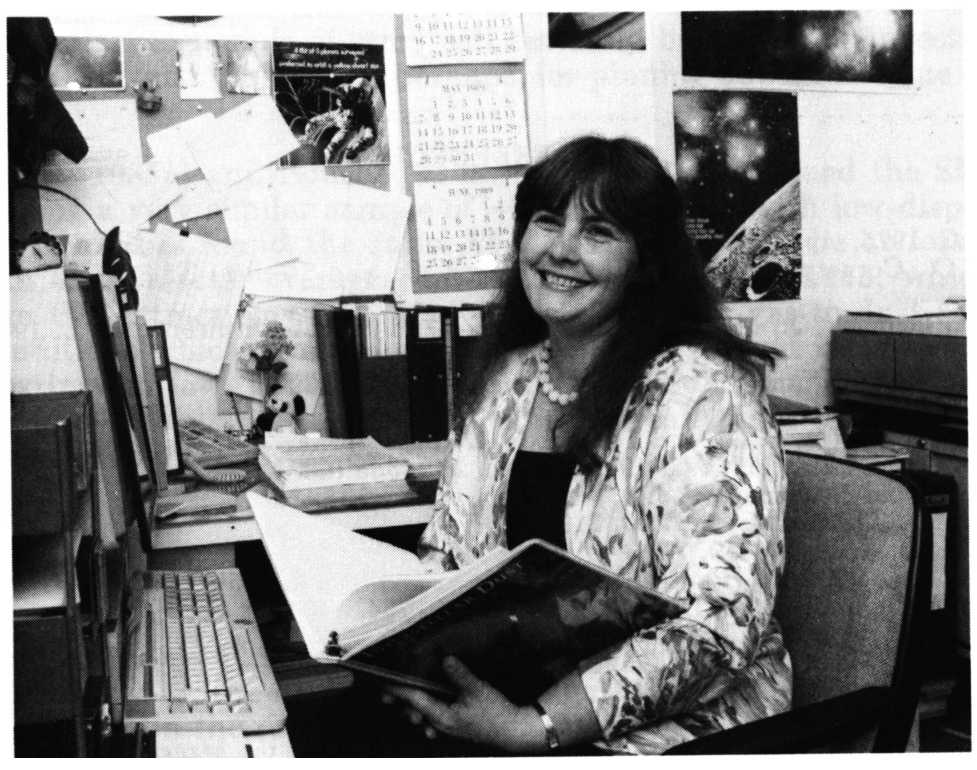

Janice Varney 